\title{
Humerus Nailing in Lateral Position (Operative Technique)
}

\author{
Wasudeo Gadegone*, Piyush Gadegone, Vijayanand Lokhande \\ GMC Chandrapur, India \\ Email: ^gadegone123@yahoo.co.in
}

How to cite this paper: Gadegone, W., Gadegone, P. and Lokhande, V. (2020) Humerus Nailing in Lateral Position (Operative Technique). Open Journal of Orthopedics, 10, 33-41.

https://doi.org/10.4236/ojo.2020.102006

Received: December 27, 2019

Accepted: February 2, 2020

Published: February 5, 2020

Copyright (อ 2020 by author(s) and Scientific Research Publishing Inc. This work is licensed under the Creative Commons Attribution International License (CC BY 4.0).

http://creativecommons.org/licenses/by/4.0/ (c) (i) Open Access

\begin{abstract}
With the advent of good designs of nails, straight and angled in the arena of treatment for fractures from surgical neck of humerus to approximately $5 \mathrm{~cm}$ above the olecranon fossa, nailing is gaining popularity in recent literature. Many different nails are available in market with different proximal and distal locking configurations. Beach chair and supine are the main principle positions for antegrade humerus nailing. Beach chair or supine is the preferred position by many, but there is always difficulty in distal locking by free hand technique as it is difficult to locate the distal locking hole due to rounded smooth anatomy of anterior distal humerus and fear of neurovascular complications in both anteroposterior and latero-medial locking. The aim of this article is to demonstrate the utility of lateral position in ease of nailing all types of humerus fractures. The technique and illustrations below describe the positioning of patient, image intensifier and free hand postero-anterior distal locking without injury to neurovascular structures.
\end{abstract}

\section{Keywords}

Humerus Fracture, Interlock Nailing, Lateral Position, Antegrade Nailing

\section{Introduction}

Humeral shaft fracture has largely been treated by reduction and plaster cast followed by functional brace with good results however it has limitations in cases of nerve and extensive soft-tissue injury, multiple fractures, non-compliance or obesity [1]. The open reduction with internal by plate and screws is the gold standard of surgical treatment, but problems such as excessive soft tissue stripping, radial nerve injury and difficulty in segmental fracture are well recognized [2]. Elastic intramedullary nails and antegrade Rush nails with three-point fixation also give equally good results [3]. But recently the introduction of inter- 
locking system and varieties of the nail designs now are possible to treat humeral shaft fracture with minimal invasive surgery and comparable results with plating which requires opening and periosteal stripping with its own complications [4]. Even if open reduction requires, it is a very small incision only to expose fracture site. However, these theoretical advantages were not confirmed by the first clinical trial and severe complications such as shoulder pain, delayed union, non-union and iatrogenic fractures were reported [5]. With the growing experience in surgical technique and progress in implant design, the possibility of antegrade insertion, improved rotational stiffness have led to better results. In antegrade nailing mostly done in beach chair or supine position hence there is always difficulty in distal locking by free hand technique as it is difficult to locate the distal locking hole due to rounded smooth anatomy of anterior distal humerus and fear of neurovascular complications in both anteroposterior and latero-medial locking [6] [7]. The lateral position distal locking is a procedure by postero-anterior direction without changing the $\mathrm{C}$ arm and position of the limb. The aim of this article is to demonstrate the utility of lateral position in case of nailing all types of humerus fractures.

\section{Operative Technique}

\subsection{Patient Positioning}

Patient is placed on radiolucent orthopedic table in contralateral lateral decubitus position with 10 - 15 degree posterior sag of ipsilateral shoulder (Figure 1). Patient's body is secured with well-padded side supports over the sacrum and pubis, soft cushioning at all bony prominences and appropriate head-neck elevation for adequate airway access.

Positioning of the patient, Surgeon, assistant, image intensifier and the anesthetist with his machine at a predefined place is the key in execution of the surgery. The surgeon stands at the head end of the patient's shoulder and assistant stands opposite to the surgeon at posterior aspect of the patient holding the arm at forearm and elbow. The assistant has a pivotal role in achieving and maintaining reduction with traction and manipulation of the arm with viewing images in all planes during the procedure. Image intensifier is placed in transverse plane giving unobstructed anteroposterior view from shoulder to elbow at all times. Viewing in different planes is usually achieved by rotating the image intensifier or changing of position of shoulder by arm and elbow rotation (Figure 2).

\subsection{Procedure}

Patient is placed in the lateral position. A small $2 \mathrm{~cm}$ skin incision from the anterolateral edge of the acromion is given and subcutaneous tissue sharply incised (Figure 3). Deltoid muscle is split along the raphe followed by incising and/or partially resecting the subdeltoid bursa. The supraspinatus tendon is incised in line with its fibers with a pointed scalpel blade and both borders of the tendon 
are retracted with sutures. Expose the superior humeral head cartilage medial to the greater tuberosity.

\subsection{Entry Point}

Fracture is reduced by using traction, varus/valgus, and rotational force applied manually. Entry point is located at the lateral edge of articular surface in AP plane and at center of humeral head in lateral plane with a $3 \mathrm{~mm}$ steinman pin and confimed under $\mathrm{C}$ arm image (Figure 4).

The entry point is enlarged with bone owl and a $2.5 \mathrm{~mm}$ guide wire is passed through the correct entry point, and guide wire is passed across the fracture site (Figure 5).

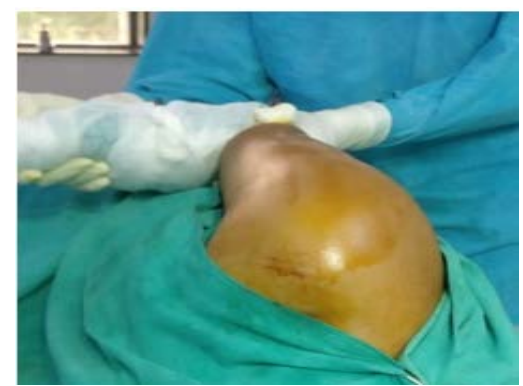

Figure 1. Lateral position.

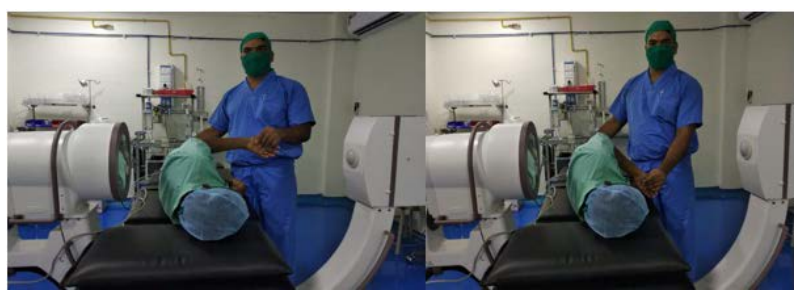

(a)

(b)
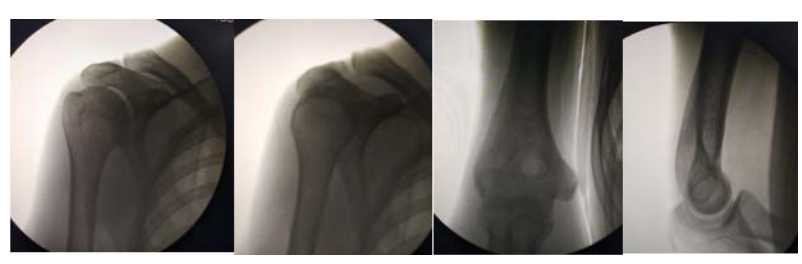

(c)

(d)

Figure 2. (a) $\mathrm{C}$ arm position; (b) Viewing in different view; (c) $\mathrm{C}$ arm image of proximal humerus; (d) C Arm image of distal humerus.

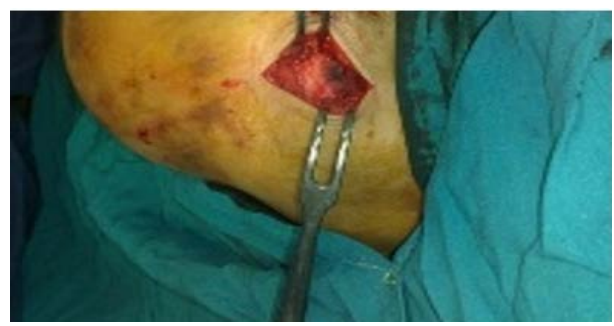

Figure 3. Anterolateral incision. 


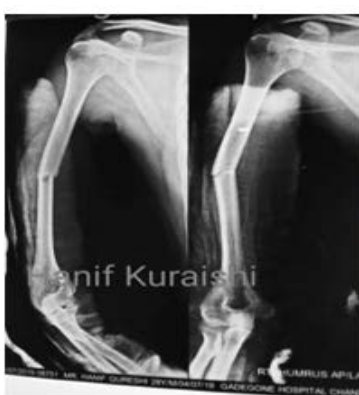

(a)

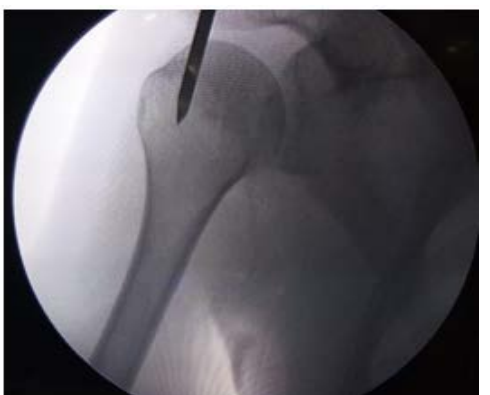

(b)

Figure 4. (a) X ray showing fracture humerus; (b) Entry point.

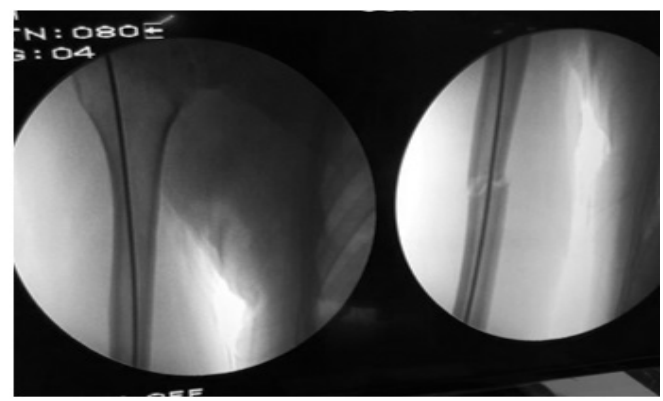

Figure 5. Reduction and guide wire insertion.

\subsection{Reaming of the Canal}

Reaming of the canal is carried out sequentially through the fracture site into the distal medullary canal up to one $\mathrm{cm}$ proximal to olecranon fossa (Figure 6).

\subsection{Insertion of Nail}

The appropriate size and the length of the humerus nail are inserted with slightly rotating movements down to the fracture line. The fracture site is firmly held by the assist to avoid movements at the fracture site which helps in the prevention of injury to the radial nerve. The nail is passed across the fracture site and final seating of the nail is done in distal metaphysis with gentle blows after accurate control of rotation. Care is taken to avoid distraction at the fracture site. Appropriate size of the nail means a nail which is embedded in the distal metaphysis and is buried at least $5 \mathrm{~mm}$ beneath the articular surface and has no distraction at fracture site (Figure 7).

\subsection{Proximal Locking}

Proximal locking is done with help of jig. Number of locking screws and their position depends on the fracture configuration and location. At least two proximal bolts are considered sufficient for shaft fractures and multiple angled locking with maximum bolts are required for proximal and metaphyseal fracture. If gapping is evident at fracture site elbow is stroked gently to achieve compression or first distal locking and extraction blows shall achieve compression at fracture site. Check the nail is sub articular and then do proximal locking (Figure 8). 


\subsection{Distal Locking}

During freehand distal locking, the patient's arm rests on his or her body and the forearm is rested on the anterior pelvic support on sterile padding. By changing the amount of padding, arm rotation can be adjusted to give a perfect view of the distal locking hole. Once distal interlock holes appear as perfect circles in AP view, a small stab incision is taken on posterior part the lower arm. The hole is located with a $3 \mathrm{~mm} \mathrm{k}$ wire and posterior cortex is perforated. The $\mathrm{K}$ wire is replaced by $3.5 \mathrm{~mm}$ drill bit and further cortex is drilled and locking is done with $3.9 \mathrm{~mm}$ appropriate length screw. Posterior surface of humerus is flat with less musculature, hence positioning of drill and locking is easy. Implant position, fracture reduction is checked in both planes and the zig removed (Figure 9).

Wounds are copiously irrigated and closed in layers. Post of images is taken to check the reduction, placement of nail and screws (Figure 10).

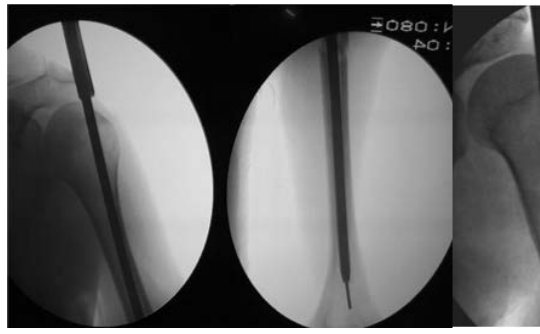

(a)

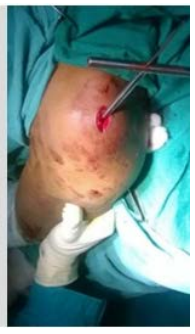

(b)

Figure 6. Sequential reaming of canal over guide wire.

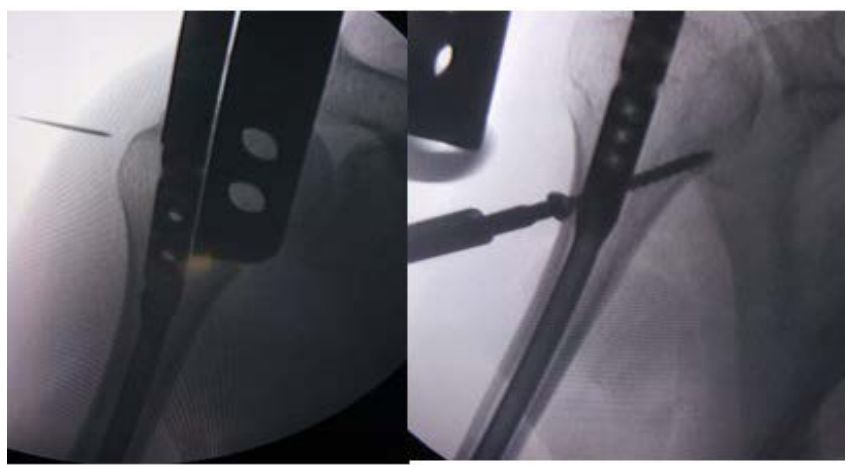

(a)

(b)

Figure 7. (a) Insertion of nail; (b) Proximal locking.

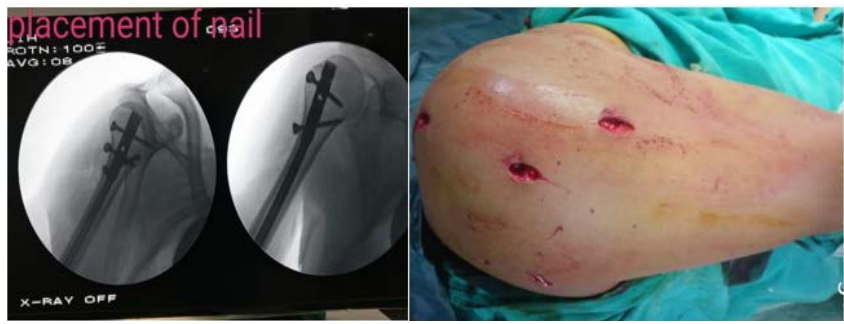

(a)

(b)

Figure 8. (a) Proximal locking; (b) Minimal incisions. 


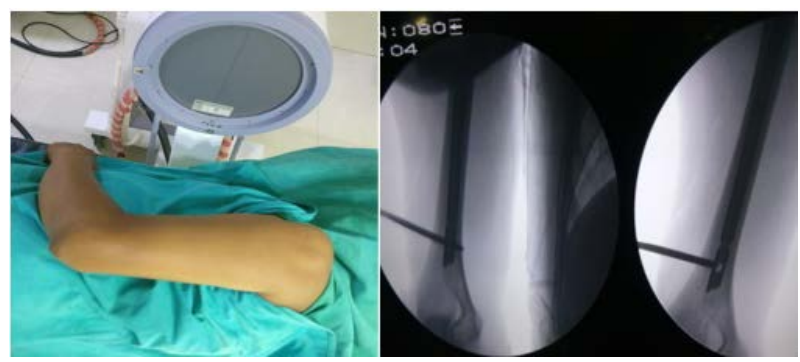

(a)

(b)

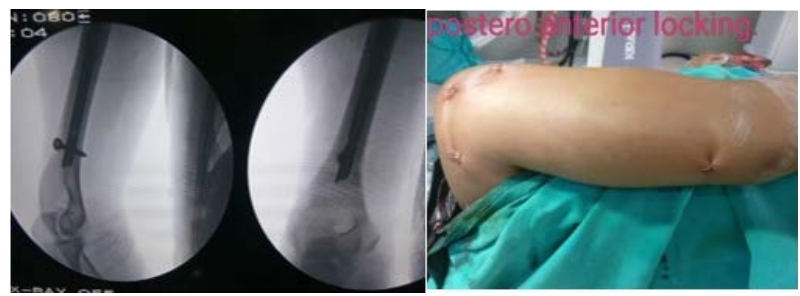

(c)

(d)

Figure 9. (a) Lateral position; (b) Drill insertion; (c) Insertion of postero anterior locking bolt; (d) Final stitch on posterior aspect of humerus.

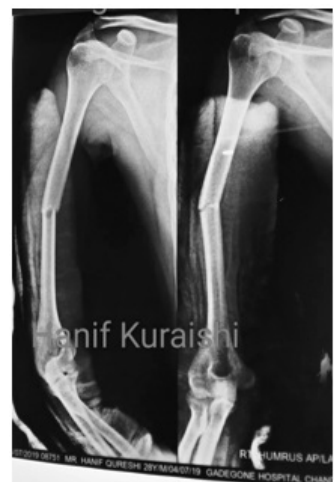

(a)

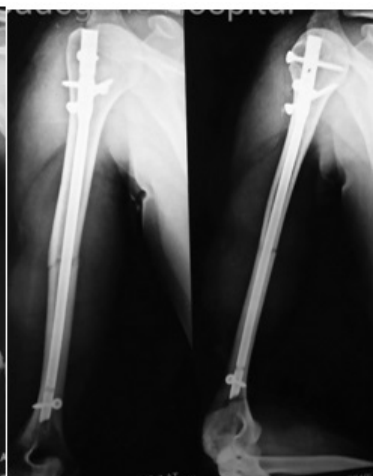

(b)

Figure 10. Diaphyseal fracture humerus, pre op and post operative image.

\section{Rehabilitation}

Immediate Post-op arm is placed in a sling pouch. Patients are encouraged to start finger, wrist and elbow movements once the anesthesia weans off. Gentle pendulum exercises, shoulder shrugs and static exercises are begun once pain free.

Case example (Figure 11)

The humeral fracture fixation with locked intramedullary nailing in lateral position offers an appealing solution.

\section{Advantages of Lateral Position}

1) Entry point location is easy extending the shoulder without changing the position of C-arm.

2) Unobstructed views from shoulder to elbow are possible without repeated change in positioning of image intensifier. 


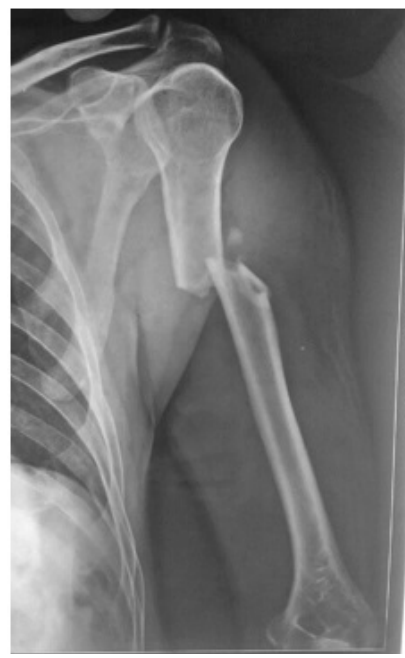

(a)

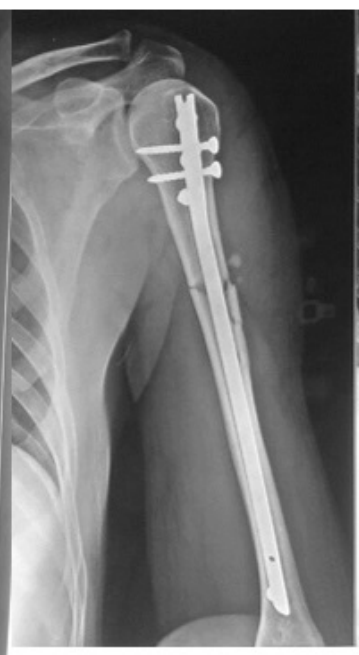

(b)

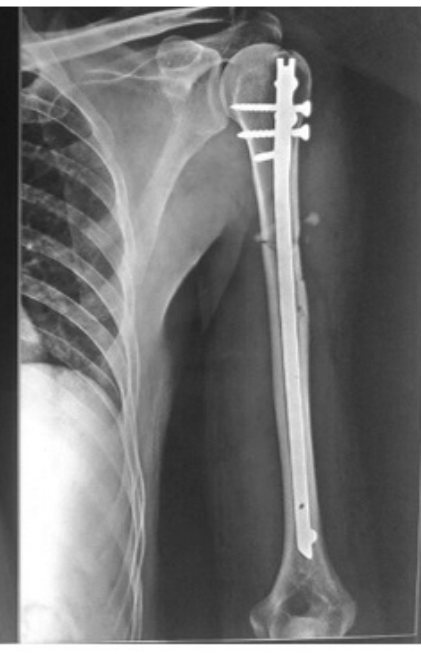

(c)

Figure 11. A pre op and B post op. Proximal humerus multilock nail.

3) Manipulation of the fracture and the limb is possible with ease.

4) Posteroanterior distal locking proves to be the biggest advantage of this position as found by easier hole location, no slippage on flat posterior surface, no risk of neurovascular damage.

5) All anterior, lateral and posterior aspects of humerus can be approached in case an open reduction is warranted.

6) Less assistance is required and low radiation exposure.

\section{Pearls and Pitfalls}

1) Entry point depends on the design of nail.

2) Incision through the supraspinatus in line with its fibers.

3) Before proximal or distal locking care must be taken for exact placement of tip of the nail sub articular.

4) No distraction at the fracture site.

5) Patient may experience tingling and numbness in a lower down contralateral limb.

6) True lateral picture is not possible in this position.

7) Firm support at the fracture site while reduction and reaming to avoid injury to radial nerve.

8) Early mobilization of shoulder to avoid adhesions and stiffness.

\section{Discussion}

The potential deleterious effects on shoulder function have been described and debated most after antegrade nailing of humerus. This can be due to impingement of proximal nail tip or proximal locking screw due to adhesive capsulitis or due to rotator cuff tears. But with small incision and identification of rotator muscle with sharp incision and reposition of nail subchondral in most of the, $80 \%-95 \%$ of patients regained their normal shoulder function and rest regained with vigourous physiotherapy [3]. Post-operative early mobilization of the 
shoulder and elbow was very critical in attaining full range of movements in these patients. Apart from this anterior-posterior and lateral-medial locking techniques have been linked to neurovascular complications including radial and lateral cutaneous nerve injuries as there is always tendency of the tip of the drill to slide and cause iatrogenic injury [6] [8].

Postero anterior distal locking there are no major neurovascular structures at risk in the posterior aspect of the arm. At this level, the posterior humerus has a flatter surface than laterally or anteriorly, reducing the tendency of the tip of the drill to slide and method has technical and safety advantages over other techniques. It reduces the duration of the hospital stay. Complications like nonunion can be avoided by intraoperative compression and avoiding distraction at fracture site.

\section{Conclusion}

Closed intramedullary interlock nailing in lateral position is a safe and reliable method of treating humeral shaft fractures. Nailing in lateral position is the least invasive surgical technique and avoids injuring the important neurovascular anterior structure. It is ideal in patients with polytrauma and osteoporosis. Early intensive physiotherapy hastens the recovery of shoulder function.

\section{Conflicts of Interest}

The authors declare no conflicts of interest regarding the publication of this paper.

\section{References}

[1] Ekholm, R., Tidermark, J., Törnkvist, H., Adami, J. and Ponzer, S. (2006) Outcome after Closed Functional Treatment of Humeral Shaft Fractures. Journal of Orthopaedic Trauma, 20, 591-596. https://doi.org/10.1097/01.bot.0000246466.01287.04

[2] Oh, C.-W., Byun, Y.-S., Oh, J.-K., Kim, J.-J., Jeon, I.-H., Lee, J.-H. and Park, K.-H. (2012) Plating of Humeral Shaft Fractures: Comparison of Standard Conventional Plating versus Minimally Invasive Plating. Orthopaedics \& Traumatology: Surgery \& Research, 98, 54-60. https://doi.org/10.1016/j.otsr.2011.09.016

[3] Gadgone, W.M. and Salphale, Y. (2008)Antegrade Pinning for Fractures of Humeral Shaft A Retrospective Analysis of 200 Cases. European Journal of Orthopaedic Surgery and Traumatology, 18, 93-99. https://doi.org/10.1007/s00590-007-0267-4

[4] Pickering, R.M., Crenshaw Jr., A.H. and Zinar, D.M. (2002) Intramedullary Nailing of Humeral Shaft Fractures. Instructional Course Lectures, 51, 271-278.

[5] Garnavos, C. (2011) Diaphyseal Humeral Fractures and Intramedullary Nailing: Can We Improve Outcomes? Indian Journal of Orthopaedics, 45, 208-215. https://doi.org/10.4103/0019-5413.67117

[6] Noger, M., Berli, M.C., Fasel, J.H. and Hoffmeyer, P.J. (2007) The Risk of Injury to Neurovascular Structures from Distal Locking Screws of the Unreamed Humeral Nail (UHN): A Cadaveric Study. Injury, 38, 954-957. https://doi.org/10.1016/j.injury.2007.04.014

[7] Blyth, M.J., Macleod, C.M., Asante, D.K. and Kinninmonth, A.W. (2003) Iatrogenic 
Nerve Injury with the Russell-Taylor Humeral Nail. Injury, 34, 227-228.

https://doi.org/10.1016/S0020-1383(01)00176-0

[8] Rupp, R.E., Chrissos, M.G. and Ebraheim, N.A. (1996) The Risk of Neurovascular Injury with Distal Locking Screws of Humeral Intramedullary Nails. Orthopedics, 19, 593-595. 